Research Article

\section{Snake envenomation during pregnancy}

\section{Denisse G Martinez and Newton G Osborne*}

Maternal and Child Hospital José Domingo De Obaldía David, Chiriquí, Republic of Panama

\section{Abstract}

Background: We report our experience with management and treatment of pregnant women who were admitted at our institution for snake envenomation.

Methods: We reviewed the charts and recorded the management, treatment, and outcome of 51 pregnant women admitted to our high-risk obstetric service with a diagnosis of snakebite.

Results: One patient experienced spontaneous abortion, 2 had intrauterine fetal death due to placental abruption, 2 delivered malformed babies, 1 had premature birth of twins with low birthweight. There were no maternal deaths.

Conclusion: Snake-venom poisoning in pregnancy is a complex medical emergency that involves complications at the site of the bite and may also involve dysfunction of multiple organ systems in both mother and fetus.

\section{More Information}

*Address for Correspondence: Newton G. Osborne, PhD, MD, Department of Obstetrics and Gynecology, Maternal and Child Hospital José Domingo De Obaldía David, Chiriquí, Republic of Panama, and Professor, University of Panama, Section 0413-00153, Boquete, Chiriquí, Republic of Panama, Tel: (507)-6611-3305: Email: newtongo@gmail.com

Submitted: 08 December 2019 Approved: 19 December 2019 Published: 23 December 2019

How to cite this article: Martínez DG, Osborne NG. Snake envenomation during pregnancy. Clin J Obstet Gynaecol. 2019; 2: 150-155.

DOI: dx.doi.org/10.29328/journal.cjog.1001039

Copyright: @ 2019 Martínez DG, et al. This is an open access article distributed under the Creative Commons Attribution License, which permits unrestricted use, distribution, and reproduction in any medium, provided the original work is properly cited.

(W) Check for updates

OPEN ACCESS

\section{Introduction}

Panama has the highest incidence of snakebites in Central America, with approximately 1,800 documented snakebite envenomations each year and a mortality rate of 0.5 per 100,000 inhabitants [1]. The viperid species Bothrops asper is widely distributed throughout our country and is responsible for the vast majority of snakebites [5]. In contrast, The United States of America with a much larger population, is estimated to have an incidence of bites by venomous snakes between 7,000 and 8,000, of which only 5 or 6 result in death [3].

According to the Ophidian Research and Response Center, there are approximately 140 snake species in Panama, of which 25 are considered to be dangerous to humans (V. Martínez Cortés, personal communication, October 5, 2019).

Different from what is reported to be the case in Southern California [4], most victims of snakebites in Panama are males between the ages of 15 to 59 [5], many victims are women, predominantly indigenous women, of whom a few are pregnant. The majority of pregnant victims of snakebites come from the comarcal areas, especially from the Ngäbe Bugle comarca, which is between the provinces of Chiriqui, Veraguas, and Bocas del Toro. They are the most common pregnant victims of snake bite due in part that women in this area frequently walk barefoot in the fields and forests, even at night time.
Most snakebites in Panama occur during the rainy season months of September and October [5].

However, as is the case in the USA [6], deaths from snake bite envenomation at any time of the year typically occur in children, in the elderly, and in victims to whom antivenom is not given, is given after a delay, or is delivered in insufficient quantities.

This article focuses on the management and treatment of venomous snakebites of pregnant women in the provinces of Chiriqui, Bocas del Toro, and the Ngäbe Bugle comarca of the Republic of Panama; however, the principles of management apply worldwide to pregnant patients who are victims of poisonous snake bites.

\section{Methods}

We carried out a retrospective, descriptive, cross-sectional study conducted at the Hospital Materno Infantil José Domingo de Obaldia (HMIJDDO), in the City of David, Province of Chiriquí. We used as data source the clinical history of patients admitted through the emergency department with a diagnosis of snakebite during the period between January 1 , 2008 and December 31, 2018.

A data collection sheet made for this purpose was used, which included number of cases per year, victim's age group, number of previous pregnancies, personal medical 
history, the time, season of the year, and gestational age when the snakebite occurred. We also recorded the snake's identification, the anatomical region of the bite, and the local and systemic effects the patients presented with at the time of admission to the emergency room. We then recorded the amount of antivenom serum vials used and time when administration started, if treatment was indicated.

We recorded whether there were evidences of adverse reaction to the antivenom, if there was any use of antibiotics, the number of days in the hospital following the event, and the final outcome of the pregnancy, which we defined as good, if there was delivery of a healthy, full-term baby, and other, if intrauterine fetal death, premature birth, low birth weight, fetal malformation, or spontaneous abortion was diagnosed following snake envenomation.

Data were tabulated in tables using Word Access $2016^{\circledR}$ and Microsoft Office Excel, for comparison and subsequent analysis, using frequency measurements and percentages for the variables. For the bivariate analysis, the statistical analysis software of IBM SPSS version 24 was used and the Chi-square test was performed, taking the value of $p<0.05$ and a $95 \%$ confidence level as statistically significant.

The study protocol was approved by the hospital's ethics and investigation committee. The authors asume full responsibility for the accuracy and completeness of the data presented and the result analyses.

\section{Results}

Of 51 patients admitted to the Obaldía Women and Children Hospital (HMIJDDO) with a snakebite diagnosis. One pregnant woman admitted for a skin autograft had a history of snakebite that was treated four months prior to the admission for the skin graft. As it was a case of a non-recent bite, she was excluded from the study, which resulted in a total of 50 patients whose files were recorded in the data collection form parentheses figure 1 .

The distribution per year of pregnant patients with snakebite is shown in figure 2 , with the greatest number of cases during 2010, 2011, 2015 and 2016.

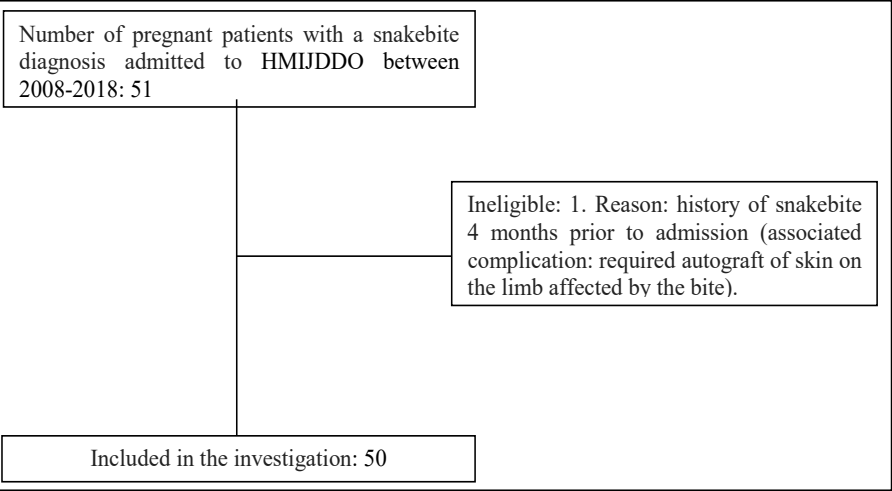

Figure 1: Flow chart of inclusionof patients to the study. Note: HMIJDDO refers to José Domingo de Obaldía Women and Children Hospital.

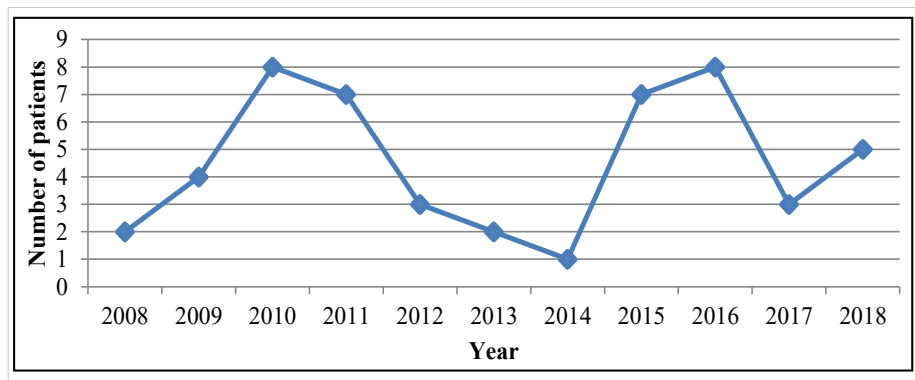

Figure 2: Distribution per year of pregnant women with snakebites. Source: Gynecology and Obstetrics Service. HMIJDDO (2008 - 2018).

A summary of the variables studied with their respective absolute and percentage frequency is in table 1 .

\section{Sociodemographic characteristics and background}

The age group of pregnant women most affected by snakebite was $21-25$ years in $40 \% .58 \%$ of the patients were multiparous, with a range of previous deliveries between $2 \%$ and $5.92 \%$ of the patients were between the second and third trimester of pregnancy at the time of the snakebite. Only two patients reported underlying medical conditions (asthma and another patient with chronic hypertension and laryngeal stenosis secondary to intubation after craniocerebral trauma).

\section{Event features}

The months in which more bite events occurred were January and September, with an increase in the number of cases during the rainy season. $80 \%$ of the patients were not able to identify the snake responsible for the bite, while in $16 \%$, identification was made verbally, that is, without bringing the snake to the hospital, and described the snake as " $\mathrm{X}$ ", fer de lance (Bothrops asper), or of "chocolate and black colors with $\mathrm{X}$-shaped spots." Two of the 50 patients did bring the snake to the hospital for objective identification by the physician.

\section{Characteristics of the lesion}

Of the 50 patients, 43 included some type of local effect after the bite, while 23 specified systemic effects. Among the local effects, they reported pain, edema and erythema in 31\%, $30 \%$ and $14 \%$ respectively; other local effects observed were local bleeding, heat at the site, bullae, ecchymosis, necrosis, paraesthesia, difficulty in mobilization, pruritus, burning sensation, and absent pulses in the affected area.

Among the systemic effects, the cardiovascular and haematological system's involvement, mainly hypertension and systemic bleeding were the most common. Only one patient had neurological sequelae, which consisted of severe spasticity and cortical blindness following a bite by an unidentified snake at 22 weeks gestation.

\section{Hospital practices}

Only one of the patients was treated with anticoral serum. The rest of the patients were treated with a polyvalent 
Table 1: Characteristics of pregnant patients with snakebite

\begin{tabular}{|c|c|c|c|}
\hline Variable & Indicator & Absolute frequency & percentual frequency \\
\hline \multirow{7}{*}{ Victim's age group } & $\leq 15$ & 1 & 2 \\
\hline & $16-20$ & 9 & 18 \\
\hline & $21-25$ & 20 & 40 \\
\hline & $26-30$ & 11 & 22 \\
\hline & $31-35$ & 6 & 12 \\
\hline & $36-40$ & 2 & 4 \\
\hline & $\geq 41$ & 1 & 2 \\
\hline \multirow{4}{*}{ Number of previous pregnancies } & Nulliparous & 8 & 16 \\
\hline & Primiparous & 7 & 14 \\
\hline & Multiparous & 29 & 58 \\
\hline & Grand multiparous & 6 & 12 \\
\hline \multirow{3}{*}{ Pregnancy trimester } & First trimester & 4 & 8 \\
\hline & Second trimester & 24 & 48 \\
\hline & Third trimester & 22 & 44 \\
\hline \multirow{3}{*}{ Underlying medical conditions } & Yes & 2 & 4 \\
\hline & No & 47 & 94 \\
\hline & Not recorded & 1 & 2 \\
\hline \multirow{2}{*}{ Period of the event } & Dry season (January to March) & 11 & 22 \\
\hline & Rainy season (April to December) & 39 & 78 \\
\hline \multirow{4}{*}{ Time of the bite } & Morning (6:00 a.m. - 12:00 p.m.) & 10 & 20 \\
\hline & Afternoon (12:00 p.m. - 6:00 p.m.) & 12 & 24 \\
\hline & Night (6:00 p.m. - 6:00 a.m.) & 25 & 50 \\
\hline & Not registered & 3 & 6 \\
\hline \multirow{3}{*}{ Snake identification } & Objective identification & 2 & 4 \\
\hline & Verbal identification & 8 & 16 \\
\hline & Not possible & 40 & 80 \\
\hline \multirow{3}{*}{ Anatomical region of the bite } & Arms & 5 & 10 \\
\hline & Legs & 44 & 88 \\
\hline & Not registered & 1 & 2 \\
\hline \multirow{3}{*}{ Local effects } & Yes & 43 & 86 \\
\hline & No & 6 & 12 \\
\hline & Not registered & 1 & 2 \\
\hline \multirow{2}{*}{ Sistemic effects } & Yes & 23 & 46 \\
\hline & No & 27 & 54 \\
\hline \multirow{7}{*}{ Amount of antivenom serum vials } & 0 & 4 & 8 \\
\hline & $1-5$ & 13 & 26 \\
\hline & $6-10$ & 19 & 38 \\
\hline & $11-15$ & 5 & 10 \\
\hline & $16-20$ & 5 & 10 \\
\hline & $\geq 21$ & 0 & 0 \\
\hline & Not registered & 4 & 8 \\
\hline \multirow{7}{*}{ Time until the placement of the antivenom serum (hours) } & $\leq 6$ & 26 & 52 \\
\hline & $7-12$ & 9 & 18 \\
\hline & $13-24$ & 6 & 12 \\
\hline & $25-48$ & 1 & 2 \\
\hline & $\geq 49$ & 0 & 0 \\
\hline & Not registered & 4 & 8 \\
\hline & Not administered & 4 & 8 \\
\hline \multirow{4}{*}{ Adverse reaction to the antivenom } & Yes & 6 & 12 \\
\hline & No & 37 & 74 \\
\hline & Does not apply & 4 & 8 \\
\hline & Not registered & 3 & 6 \\
\hline \multirow{2}{*}{ Antibiotic use } & Yes & 44 & 88 \\
\hline & No & 6 & 12 \\
\hline \multirow{3}{*}{ Days in the hospital after the event } & 1 & 7 & 14 \\
\hline & $2-5$ & 35 & 70 \\
\hline & $\geq 6$ & 8 & 16 \\
\hline & Good & 21 & 42 \\
\hline & Intrauterine fetal death & 2 & 4 \\
\hline Fetal outcome & Premature birth of low birthweight twins & 1 & 2 \\
\hline & Abortion & 1 & 2 \\
\hline & Malformation & 2 & 4 \\
\hline & Not available & 23 & 46 \\
\hline
\end{tabular}

Source: Gynecology and Obstetrics Service. José Domingo de Obaldía Women and Children Hospital (2008 - 2018). 
antivenom manufactured in Costa Rica (anti-Bothrops sp, antiCrotalus sp., anti-Lachesis sp., Instituto Clodomiro Picado) which is routinely used in Panama for the treatment of these envenomations. Of 50 patients, 6 had adverse reactions to the antivenom. Reactions to antivenom included respiratory distress, serum sickness, skin rash, nausea, vomiting and diaphoresis. The amount of antivenom vials used in these patients ranged between 5 and 15 vials. Forty four patients were treated with antibiotic therapy. Penicillin sodium and ceftriaxone (29\% and $26 \%$ respectively) were the antibiotics more commonly used.

\section{Response to treatment}

We were not able to review the birth data in $46 \%$ of the patients, because they delivered elsewhere. However, $42 \%$ of patients treated who delivered at our hospital had a good outcome, with the birth of a healthy, full-term baby. There was a $6 \%$ fetal loss, one of which was a spontaneous abortion and two others that resulted in intrauterine fetal death.

There were three patients with placental abruption. The first one received the antivenom in the first 6 hours after the bite. Because she developed lower abdominal pain and transvaginal bleeding, an emergency caesarean section was performed, with delivery of a healthy baby. The mother was found to have a blood infiltrated myometrium. The second patient was exposed to the venom without receiving an antidote for 24 hours. When she arrived at the hospital she already had a fetal death. The third patient received antivenom at the reference center 3 hours after the bite. When she arrived at the hospital the fetal heart rate was found to be less than 110 beats per minute. She was immediately prepared for caesarean section, but before incision, (22 minutes after arrival in the emergency room), it was not posible to auscultate a fetal heartbeat. The two patients with fetal death also developed compartment syndrome.

The patient who had the spontaneous abortion was 10 weeks pregnant. She had received antivenom 3 hours after the bite and was discharged from the hospital following recovery. Eleven days after the bite she developed serum sickness, and at 16 weeks gestation she was readmitted to the hospital with an inevitable abortion. In the two cases with fetal malformations, the bite had occurred at 20 and 17 weeks of gestation. The first one, gave birth to a baby with cleft lip and palate and the second one, gave birth to a baby with bilateral preauricular sinus. Prior to discharge, other commonly associated malformations were ruled out.

A chronic hypertensive mother with a twin pregnancy of 22 weeks was bitten by an unidentified snake but had no signs of envenomation. She was not treated with antivenom. She was discharged home. At 31 weeks of pregnancy, a caesarean section was performed due to eclampsia. She delivered two babies below the appropriate weight for gestational age. The children were again seen at our hospital when they were three years old and were diagnosed with chronic malnutrition and delayed psychomotor development.

\section{Bivariate analysis}

When reviewing gestational age when the snake bite took place and amount of vials of antivenom serum used, it was observed that there were a few pregnant women who were between the second and third trimester and did not merit the use of antivenom, while there were others in this same range who used a maximum of 20 vials. There was no relationship between gestational age when the bite took place and the amount of antivenom serum vials (Table 2).

\section{Discussion}

The effects of snake venom on pregnancy have not been fully elucidated. There is evidence that snake venom may cross the placenta affecting the fetus even without evidence of envenomation in the mother $[4,5]$.

The effect of snake envenomation on mother-fetus is discussed in a series of isolated case reports, with no specific management recommended [6-12]. Our greatest advantage regarding experience in this area is our being in a tropical country that affords a growing number of cases to evaluate and treat.

According to data from the Panamanian Ministry of Health, there was greater morbidity due to snake bite in general during the years 2010 and 2015. In our study, the years with the highest number of accidents in pregnant women were 2010, 2011, 2015 and 2016.

Table 2: Association of gestational age by trimester and the amount of antivenom serum vials used.

\begin{tabular}{|c|c|c|c|c|c|c|c|c|c|c|c|c|}
\hline \multicolumn{13}{|c|}{ Cross table Trimester* amount of antivenom serum vials } \\
\hline & & & \multicolumn{9}{|c|}{ Vials Used } & \multirow{2}{*}{ Total } \\
\hline & & & 0 & 1 & 3 & 5 & 7 & 8 & 10 & 15 & 20 & \\
\hline \multirow{6}{*}{ 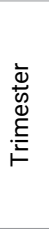 } & \multirow{2}{*}{ First Trimester } & Count & 0 & 0 & 0 & 1 & 0 & 0 & 2 & 1 & 0 & 4 \\
\hline & & $\%$ Vials Used & $0.0 \%$ & $0.0 \%$ & $0.0 \%$ & $10.0 \%$ & $0.0 \%$ & $0.0 \%$ & $12.5 \%$ & $20.0 \%$ & $0.0 \%$ & $8.7 \%$ \\
\hline & \multirow{2}{*}{ Second Trimester } & Count & 1 & 1 & 1 & 5 & 1 & 1 & 7 & 2 & 3 & 22 \\
\hline & & $\%$ Vials Used & $25.0 \%$ & $100.0 \%$ & $50.0 \%$ & $50.0 \%$ & $100.0 \%$ & $50.0 \%$ & $43.8 \%$ & $40.0 \%$ & $60.0 \%$ & $47.8 \%$ \\
\hline & \multirow{2}{*}{ Third Trimester } & Count & 3 & 0 & 1 & 4 & 0 & 1 & 7 & 2 & 2 & 20 \\
\hline & & $\%$ Vials Used & $75.0 \%$ & $0.0 \%$ & $50.0 \%$ & $40.0 \%$ & $0.0 \%$ & $50.0 \%$ & $43.8 \%$ & $40.0 \%$ & $40.0 \%$ & $43.5 \%$ \\
\hline & \multirow{2}{*}{ Total } & Count & 4 & 1 & 2 & 10 & 1 & 2 & 16 & 5 & 5 & 46 \\
\hline & & $\%$ Vials Used & $100.0 \%$ & $100.0 \%$ & $100.0 \%$ & $100.0 \%$ & $100.0 \%$ & $100.0 \%$ & $100.0 \%$ & $100.0 \%$ & $100.0 \%$ & $100.0 \%$ \\
\hline
\end{tabular}

Source: Gynecology and Obstetrics Service. José Domingo de Obaldia Women and Children Hospital (2008 - 2018$).$ 


\begin{tabular}{|c|c|c|c|}
\hline \multicolumn{4}{|c|}{ Chi-square test } \\
\hline & Value & df & Bilateral asymptotic significance \\
\hline Pearson's Chi-square & $6,066^{\mathrm{a}}$ & 16 & 0.987 \\
\hline Likelihood ratio & 7.655 & 16 & 0.958 \\
\hline Linear association & 0.305 & 1 & 0.581 \\
\hline No of valid cases & 46 & & \\
\hline
\end{tabular}

A 2002 case series of 39 pregnant women with snake poisoning in Sri Lanka had a 30\% fetal loss rate and there were fetal malformations (hydrocephalus and polydactyly) in one of the poisonings that occurred at 16 weeks gestation [7]. A worldwide review of snakebite in pregnancy from 1966 to 2009 showed a $4 \%$ maternal mortality rate and $20 \%$ fetal loss among 213 cases [11]. Another 1992 review of 50 cases of snakebites during pregnancy in the United States, reported a maternal mortality rate of $10 \%$ and a fetal death rate of $43 \%$ [9].

In contrast to the high rate of fetal loss found in previous reports, our study found $6 \%$ fetal loss. Although we had no cases of maternal death, we had a case with neurological sequelae and the case we excluded that required skin autograft.

Histopathological findings in the placenta have revealed foci of necrosis and extensive vascular congestion [5]. Several mechanisms have been proposed to explain fetal loss resulting from snake envenomation. They include fetal anoxia due to maternal shock following envenomation, a direct effect of the venom on the fetus, hemorrhages into the placenta and uterine wall causing placental abruption, premature uterine contractions initiated by the venom, pyrexia and cytokine release after tissue damage, maternal hemorrhage with acute fetal anemia and maternal anaphylaxis secondary to acute antivenom allergy [8,11-13].

There are reports of placental abruption following envenomation, the main reason for fetal death folowing maternal envenomation [14].

If the toxin in the snake venom is a coagulating agent, when it reaches the deciduo-placental cleavage zone, it may start a dissociation that ends up in placental abruption [15].

Satish, [16] describes the case of a 25-year-old patient at 20 weeks of gestation bitten by a snake. She received 7 vials of antivenom and then developed acute renal injury, managed with dialysis with gradual recovery of renal function. At 30 weeks the diagnosis of type I fetal growth restriction was made and at 32 weeks she went into preterm labor giving birth to a 1400 gram baby boy without congenital anomalies. Both mother and son were discharged in good health fourteen days later.

Malformations have been described like the delivery of a baby with hydrocephalus and polydactyly from a woman who was bitten in her 16th week of pregnancy and another newborn with hydrocephalus of a woman bitten in her $12^{\text {th }}$ week of pregnancy [13].
Though the cause-effect relationship between envenomation and malformations in these cases is unknown, it is posible that snake venom has embryotoxic and teratogenic effects. Animal studies have shown snake envenomation to cause anomalies like cleft palate, facial deformities, hepatic and myocardial damage, embryonic deaths and fetal resorption during early organogenesis [13].

This study highlighted hypertension as an episodic event without being persistent in the rest of the hospitalization. Indeed, Viperidae and Elapidae venom have cardiotoxic effects that can induce autonomic deregulation and catecholamine release, potentially leading to transient hypertension [17].

Only half of the patients in the group we reviewed received antivenom serum within the first 6 hours of the bite, which is considered to be the ideal time to prevent adverse effects. Although there is no evidence that the use of prophylactic antibiotic changes the incidence of infection, almost all patients were administered antibiotics.

From the outcomes and consequences of snakebite on pregnancy, it is clear that the three cases of placental abruption may be secondary to the snake's venom, two of them resulting in fetal death. Exposure to snake venom during pregnancy is probably unrelated to the two cases of fetal malformations that we observed. It is also doubtful that the case of premature twin birth with both babies of low birthweight for gestational age is related to the snakebite, since it is well known that multifetal pregnancy is a risk factor of growth restriction. The mother in this case had in addition a hypertensive disorder of pregnancy, which is also known to be associated with defective placental perfussion and consequently, with fetal growth restriction. In one case, spontaneous abortion occurred 6 weeks after the bite, so there is probably no cause-effect relationship in this case either. Otherwise, two of the three cases with placental abruption received the antivenom serum within 6 hours of the event, suggesting the possibility of a high utero-placental toxicity of snake venom.

\section{Conclusion}

The approach of a pregnant woman bitten by a snake should include both the general management offered to any patient who is a victim of snakebite, but in a pregnant patient, it should also generate a concern for a possible association with placental abruption, with spontaneous abortion, and with fetal malformations.

It is important to find out whether there is a direct relationship of snake envenomation with placental abruption and fetal death by further exploring the probability that the venom of some snakes may have a lethal effect on the fetus, even with timely treatment of the mother. The reported relationship between snake poison and fetal malformations [13], makes us wonder if snake venom is teratogenic, since an association of snake envenomation and malformations has been observed in animal studies. 


\section{References}

1. Gutiérrez JM. Envenenamientos por mordeduras de serpientes en América Latina y el Caribe: una visión integral de carácter regional. Bol Malariol Salud Amb. 2011; 51: 1-16.

2. Vélez $S M$, Salazar $M$, Acosta de Patiño H, Gómez L, Rodriguez A, et al. Geographical variability of the venoms of four populations of Bothrops asper from Panama: toxicological analysis and neutralization by a polyvalent antivenom. Toxicon. 2017; 132: 55-61.

PubMed: https://www.ncbi.nlm.nih.gov/pubmed/28392273

3. Gold BS, Dart RC, Barish RA. Bites by venomous snakes. N Engl J Med. 2002; 347: 347-356.

PubMed: https://www.ncbi.nlm.nih.gov/pubmed/12151473

4. Wingert WA, Chan L. Rattlesnake bites in Southern California and rationale for recommended treatment. West J Med .1988; 148: 37-44. PubMed: https://www.ncbi.nlm.nih.gov/pmc/articles/PMC1026007/

5. Pecchioa M, Suárez JA, Hessec $S$, Hershd AM, Gundackere ND. Descriptive epidemiology of snakebites in the Veraguas province of Panama, 2007-2008. Trans R Soc Trop Med Hyg. 2018; 112: 463-466. PubMed: https://www.ncbi.nlm.nih.gov/pubmed/30165536

6. Gold BS, Wingert WA. Snake venom poisoning in the United States: a review of therapeutic practice. South Med J. 1994; 87: 579-589. PubMed: https://www.ncbi.nlm.nih.gov/pubmed/8202764

7. Saade GR, Foley MR, Phelan JP y Dildy GA. Critical Care Obstetrics. USA: Wiley. 2010; 550.

8. Bergillo F, Rivas MA. Toxinología clínica. Lesiones por picaduras y mordeduras de animales. Bubok Publishing. 2013; 531.

9. Acosta H. Programa Nacional de Ofidismo y Escorpionismo. Perspectivas hacia la respuesta nacional. Panamá. [Internet]. 2017 [cited 2019 March 15]: https://www.senacyt.gob.pa/wp-content/ uploads/2018/07/Ofidismo-y-Escorpionismo-Hildaura-Acosta.pdf
10. Seneviratne $S L 1$, de Silva $C E$, Fonseka MM, Pathmeswaran $A$, Gunatilake SB, et al. Envenoming due to snake bite during pregnancy. R Soc Trop Med Hyg. 2002; 96: 272-274.

PubMed: https://www.ncbi.nlm.nih.gov/pubmed/12174776

11. Langley RL. Snakebite during Pregnancy: A Literature Review. Wilderness Environ Med. 2010; 21: 54-60.

PubMed: https://www.ncbi.nlm.nih.gov/pubmed/20591355

12. Dunnihoo DR, Rush BM, Wise RB, Brooks GG, Otterson WN. Snake bite poisoning in pregnancy. A review of the literature. J Reprod Med 1992; 37: 653-658.

PubMed: https://www.ncbi.nlm.nih.gov/pubmed/1522574

13. Padmaja DA. Snake bite in pregnancy-case report and review of literature. Int J Pregn \& Chi Birth. 2017; 2: 21-23.

14. Hanprasertpong J, Hanprasertpon T. Abruptio placentae and fetal death following a Malayan pit viper bite. J Obstet Gynaecol Res. 2008; 34: 258-261

PubMed: https://www.ncbi.nlm.nih.gov/pubmed/18412793

15. Mittal R, Dhiman B, Sood N, Sood S. A rare case of pregnancy complicated by snake bite. Int J Reprod Contracept Obstet Gynecol. 2016; 5: 243-245.

16. Satish H, Jayachandran S, Priyamvada PS, Keepanasseril A, Sravankumar J, et al. Snakebite-induced acute kidney injury requiring dialysis in second trimester of pregnancy: Successful outcomes in a therapeutic quagmire. Saudi J Kidney Dis Transpl. 2017; 28: 437-440. PubMed: https://www.ncbi.nlm.nih.gov/pubmed/28352037

17. Nelwan E, Adiwinata R, Handayani S, Rinaldi I. Severe coagulopathy and transient hypertension following a Rhabdophissubminiatus bite: a case report. Revista da Sociedade Brasileira de Medicina Tropical. 2016; 49: 520-522.

PubMed: https://www.ncbi.nlm.nih.gov/pubmed/27598644 